\title{
Localized fibrous mesothelioma of the mediastinum devoid of pleural connections
}

\author{
Moshe Balassiano, Nira Reichert, Yehudith Rosenman, Emil Hertcheg, Yair \\ Lieberman and Alon Yellin
}

Departments of Thoracic Surgery, Pulmonary Medicine, Diagnostic Radiology, and Pathology, The Chaim Sheba Medical Center, Tel Hashomer, and the Tel Aviv University Sackler School of Medicine, Israel.

\begin{abstract}
Summary: Localized fibrous mesothelioma of the pleura is an uncommon neoplasm. Very rarely (there have been three previous cases) it may occur as an isolated mediastinal tumour. Such a tumour was present for at least 3 years in a 47 year old man, causing shortness of breath and superior vena cava syndrome. A mass weighing over $600 \mathrm{~g}$ was completely resected through a median sternotomy. The pertinent literature is reviewed.
\end{abstract}

\section{Introduction}

Localized fibrous tumours originating from the pleural or the peritoneal surfaces are less common than the diffuse malignant type. ${ }^{1}$ They originate either from the mesothelial layer or the fibroblastic submesothelial area, ${ }^{2,3}$ or as recent studies suggest, from submesothelial multipotential cells. ${ }^{4}$ This type of neoplasm has received a variety of names, including localized fibrous tumour of the pleura, localized mesothelioma, fibrous mesothelioma, benign mesothelioma, and subpleural fibroma, thus reflecting the versatility of the pathological and clinical characteristics, its benign nature, as well as the controversial theories of its histogenesis.

Localized pleural mesotheliomas, usually attached to the visceral pleura, may occasionally arise from the parietal pleura, and very infrequently may extend medially, appearing as a mediastinal mass. ${ }^{5,6} \mathrm{We}$ hereby report a case of a giant mediastinal fibrous mesothelioma, apparently isolated from the pleura.

\section{Case report}

A 47 year old man was admitted in October 1986 for evaluation of a mediastinal mass discovered on a routine chest $\mathrm{X}$-ray examination 3 years earlier, at which time the patient refused further studies. During most of this period he was free of complaints,

Correspondence: Alon Yellin, M.D., Department of Thoracic Surgery, The Chaim Sheba Medical Center, Tel Hashomer 52621, Israel.

Accepted: 7 April 1989 experiencing exertional dyspnoea only several weeks $\stackrel{\frac{\Upsilon}{7}}{\frac{7}{7}}$ prior to his admission.

Physical examination revealed fullness of the nect, with venous congestion and slight deviation of tie trachea to the right. Expiration was prolonged and wheezy. The chest $\mathrm{X}$-ray showed a huge mass occup ing the anterior mediastinum, and displacing the various structures to the right (Figure 1). This was confirmed by computed tomographic scan (Figure 2), that also demonstrated that the mass was separated $\varnothing$ from the lung, aorta, and other organs. An ${ }^{131} \mathrm{I} \overrightarrow{\vec{O}}$ radionuclide scan revealed a normal thyroid, with no 3 evidence of substernal extension.

A left parasternal mediastinotomy was performed through the second intercostal space, revealing a solid $\overline{-}$ anterior mediastinal mass that bled easily. A frozen section examination confirmed the presence of viable $\overline{0}$ tissue, but a definite diagnosis could not be established.

A median sternotomy was then performed. A large bilobular mass was seen occupying almost the entire 옥 mediastinal space from the anterior chest wall to the $>$ left paravertebral area. The mass extended into the neck anteriorly to the aortic arch. The arch, along with $N$ the innominate and right subclavian arteries, was ${ }^{\circ}$ displaced posteriorly, whereas the left common $\tilde{N}$ carotid artery was lifted by the mass. The trachea and $N$ superior vena cava deviated to the right, and the left innominate vein was extremely stretched and comp-o

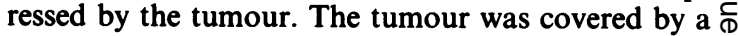
very vascular lining, which seemed to be composed of $\stackrel{\oplus}{?}$ mediastinal tissue rather than belonging to the mass 0 itself. The mediastinal pleura was intact bilaterally, $\frac{\mathrm{O}}{\mathrm{P}}$ 


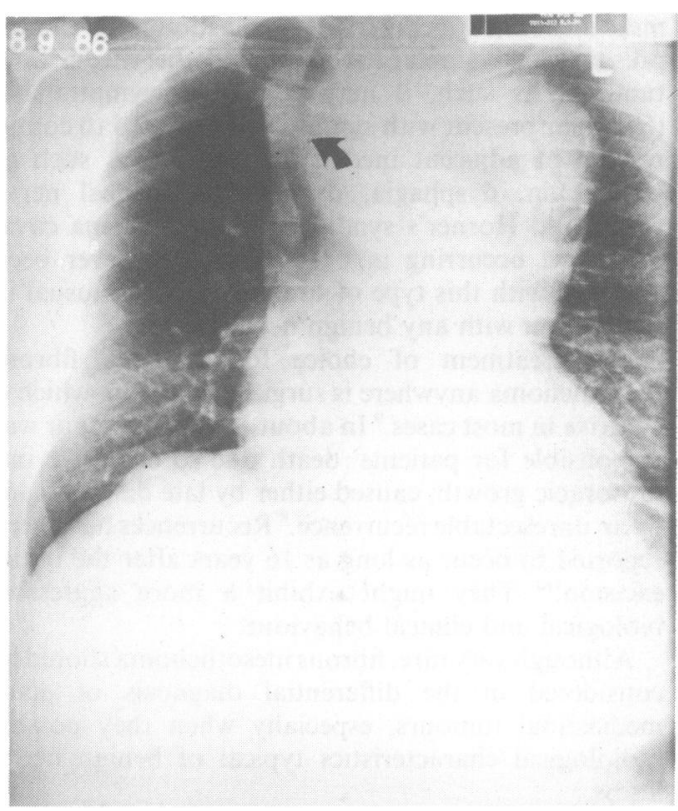

Figure 1 Chest X-ray shows a huge mediastinal mass. The mass projects mainly to the left, and displaces the trachea to the right. The tracheal lumen is compressed several $\mathrm{cm}$ above the carina (arrow).

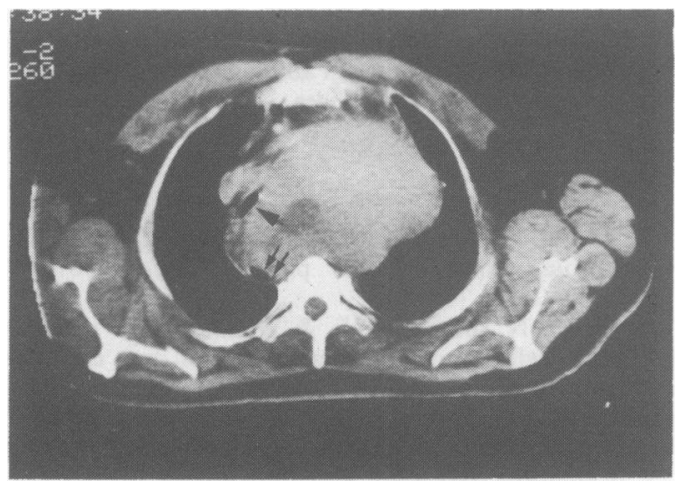

Figure 2 Computed tomography above the aortic arch. A solid mediastinal mass with areas of lucency. The trachea (arrow) and oesophagus (double arrow) are displaced to the right. The tracheal lumen is severely narrowed.

and was not connected to the tumour at any point.

With extreme caution the mass was sharply and bluntly dissected free, and removed in toto. The mediastinal pleural surfaces remained intact bilaterally.

On macroscopic examination the mass was encap- sulated, measuring $16 \times 10 \times 8 \mathrm{~cm}$, and weighing $632 \mathrm{~g}$. The outer contour was smooth, and the cut surface had a whitish-grey colour. Some areas were darker and necrotic, and a few cysts were also seen. Microscopic sections showed a spindle cell neoplasm composed of rather uniform cells, with scant cytoplasm, and ill-defined cell borders. Mitoses were rare, and there was no necrosis (Figure 3). The tumour showed different aspects. In some areas there were many thick walled vessels, or fibrosis; in others it was rich in cells that exhibited a storiform pattern.

As the nature of the tumour could not be clarified, the slides were sent to the Armed Forces Institute of Pathology in Washington, D.C., USA, where a diagnosis of localized fibrous mesothelioma was established.

The postoperative course was uneventful, except for a left vocal cord paralysis. Six months after resection the patient is asymptomatic, and the neck swelling has regressed completely.

\section{Discussion}

Primary neoplasms of the mediastinum are being recognized with increasing frequency due to improved imaging techniques. Neurogenic tumours, thymomas, lymphomas, and germ cell tumours account for almost $85 \%$ of all cases. ${ }^{7}$ A variety of mesenchymal tumours, lipoma being the most common, may arise in the mediastinum, and as a group they constitute less than $7 \%$ of all primary mediastinal tumours. Other mesenchymal tumours that have been reported less frequently are: liposarcoma, fibroma, fibro-sarcoma, myxoma, xanthogranuloma, leiomyosarcoma, rhabdomyosarcoma, and several neoplasms of vascular and lymphatic origin.

Mediastinal mesotheliomas have also been reported, usually as the diffuse malignant type. ${ }^{8-10}$ The

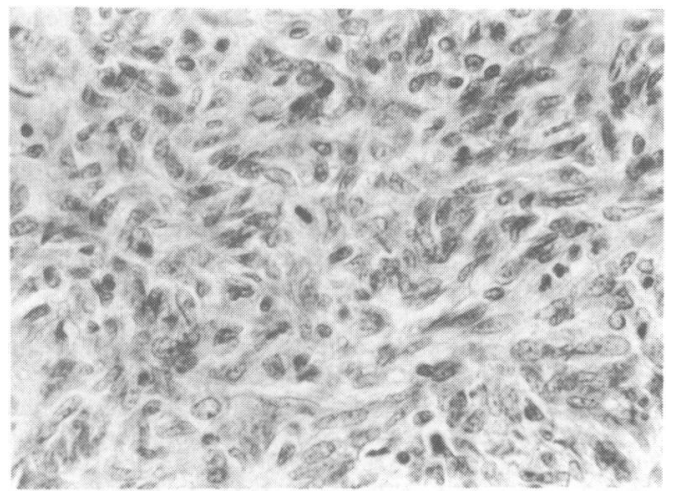

Figure 3 Spindle cell rich tumour. Mitotic figure in the centre $(\mathrm{H} \& \mathrm{E} \times 252)$. 
localized benign tumour is very rare in this location. We performed a computerized survey of the literature that revealed only one case, reported in a Polish publication. ${ }^{5}$ Reading very carefully the 20 largest series of mediastinal tumours ${ }^{6}$ accounting for close to 4000 cases we found two additional cases, included among the huge Mayo Clinic series. ${ }^{6}$ As expected, the latter were only briefly mentioned.

Mediastinal fibrous mesothelioma, although bearing a similar name to malignant mesothelioma and occurring in the same organs, is commonly believed to originate from multipotential cells in the submesothelial layer, ${ }^{4}$ and not from mesothelial cells, as previously suggested.

Approximately $80 \%$ of all localized pleural mesothelioma originate from the visceral pleura, and $20 \%$ from the parietal. Usually the tumours grow toward and occupy the interpleural space. Only on rare occasions do they extend into the chest wall or mediastinum, which contain tissues that are less compressible. In such cases the tumour is attached, at least in one point, to the pleura. Such an attachment was not observed in our case. A pleural fibrous mesothelioma can extend into, or appear as, a pulmonary parenchymal tumour. ${ }^{11}$ Several cases of intrapleural localized fibrous mesothelioma without pleural connection have also been reported. ${ }^{12,13}$

Fibrous mesotheliomas vary considerably in size, from one $\mathrm{cm}$ in diameter up to more than $3 \mathrm{~kg}$ in weight. The average diameter is about $11 \mathrm{~cm}$. They usually occur in adults, and are found equally in men and in women. ${ }^{8}$

In those rare cases where localized fibrous

\section{References}

1. Antman, K.H. Clinical presentation and natural history of benign and malignant mesothelioma. Semin Oncol 1981, 8: 313-322.

2. Osamura, R.J.Y. Ultrastructure of localized fibrous mesotheliomas of the pleura: Report of a case with histogenetic considerations. Cancer 1977, 39: 139-142.

3. Alvarez-Fernandez, E. \& Diez-Nau, M.D. Malignant fibrosarcomatous mesothelioma and benign pleural fibroma in tissue culture. Cancer 1979, 43: 1658-1663.

4. Doucet, J., Dardick, I., Singley, J.R., Van Nostrand, V.P., Bell, M.A. \& Kahn, H.J. Localized fibrous tumour of serosal surfaces: immunohistochemical and ultrastructural for a type of mesothelioma. Virchows Arch (Pathol Anat) 1986, 409: 349-363.

5. Przystasz, T., Badowski, A. \& Dumanski, Z. A case of localized pleural mesothelioma simulating mediastinal tumor. Pneumonol Pol 1984, 52: 393-396.

6. Wychulis, A.R., Payne, W.S., Clagett, O.T. \& Woolner, L.B. Surgical treatment of mediastinal tumors. $J$ Thorac Cardiovasc Surg 1971, 62: 379-391.

7. Silverman, N.A. \& Sabiston, D.C., Jr. Primary tumors and cysts of the mediastinum. In: Current Problems in Cancer. Year Book Medical Publishers, Chicago, 1977. mesothelioma appears in the mediastinum, its clinical presentation is similar to that of any other mediastinal $a$ tumour. As such, it may be either asymptomatic $\subseteq$ $(50 \%)$, or present with symptoms attributed to comp- $\overrightarrow{\bar{B}}$ ression of adjacent mediastinal structures, such as $\overrightarrow{0}$ chest pain, dysphagia, dyspnoea, laryngeal nerve $\frac{\bar{\sigma}}{\sigma}$ palsy, and Horner's syndrome. Superior vena caval $\overline{\bar{c}}$ syndrome occurring in our patient has never been $\vec{\varnothing}$ reported with this type of tumour, and is unusual in association with any benign neoplasm.

The treatment of choice for localized fibrous $\overrightarrow{0}$ mesothelioma anywhere is surgical resection, which is $\overrightarrow{-}$ curative in most cases. ${ }^{9}$ In about $12 \%$ the tumour was $\vec{\omega}^{\circ}$ responsible for patients' death due to extensive int- $\overrightarrow{0}$ rathoracic growth, caused either by late diagnosis, or $\frac{0}{3}$ by an unresectable recurrence. ${ }^{8}$ Recurrences have been reported to occur as long as 16 years after the initial on excision. $^{10}$ They might exhibit a more aggressive ${ }^{\circ}$ biological and clinical behaviour.

Although very rare, fibrous mesothelioma should be considered in the differential diagnosis of giant 0 mediastinal tumours, especially when they possess? radiological characteristics typical of benign neoplasms.

\section{Acknowledgements}

We are indebted to L. Hochholzer, M.D., R.J. Sbasching M.D., D.K. Keffner, M.D., and R.R. McKeekin, M.D. from the Armed Forces Institute of Pathology, Washington, D.C., for the examination of the slides.

8. Briselli, M., Mark, E.J. \& Dickersin, G.R. Solitary fibrous tumors of the pleura. Cancer 1981, 47: 2678-2689.

9. Okike, N., Bernatz, P.E. \& Woolner, L.B. Localized mesothelioma of the pleura: benign and malignant variants. $J$ Thorac Surg 1978, 75: 363-372.

10. Utley, J.L., Parker, J.C., Hahn, R.S. et al. Recurrent benign fibrous mesothelium of the pleura. $J$ Thorac Cardiovasc Surg 1973, 65: 830-834.

11. Dalton, W.T., Zolliker, A.S., McCaughy, W.T.E., Jacques, J. \& Kannerstein, M. Localized primary tumors of the pleura. Cancer 1979, 44: 1465-1475.

12. Abrahamson, J.R \& Friedman, N.B. Intrapulmonary $\mathrm{N}$ stromal mesothelioma. J Thorac Cardiovasc Surg 1966, 51: $300-306$.

13. Yousem, S.A. \& Flynn, S.D. Intrapulmonary localized fibrous mesothelioma. Am J Clin Pathol 1988, 89: $365-369$. 